\title{
Angiosarcoma of the Breast with Silicone Granuloma: A Case Report
}

\author{
MIKI TAKENAKA, MAKI TANAKA, MAKOTO ISOBE, RIN YAMAGUCHI*, \\ MASAMICHI KOJIRO* AND KAZUO SHIROUZU** \\ Department of Surgery, Social Insurance Kurume Daiichi Hospital, Kurume 830-0013 and \\ Departments of Pathology* and Surgery**, Kurume University School of Medicine, \\ Kurume 830-0011, Japan
}

Received 15 April 2009, accepted 19 August 2009

Edited by MINORU YAGI

\begin{abstract}
Summary: Angiosarcoma of the breast is a rare non-epithelial tumor and that accounts for less than $0.1 \%$ of primary malignancies of the breast. The disease has a relatively higher occurrence among young people, and its prognosis (3-year-survival of only 38\%) is extremely poor compared to breast cancer. Here we present a case of an 87-year-old woman who had undergone bilateral breast augmentation with silicone injections in her youth. Although she became aware of a tumor in her right breast, she waited 8 years before seeking treatment. She felt the tumor growing and experienced swelling and pain, but she ended up declining therapy at that time. Two years later she was brought to our hospital by ambulance for continuous bleeding from the same tumor of the breast, which by that time was over $11 \mathrm{~cm}$ in diameter. We performed emergency mastectomy. The histological diagnosis was angiosarcoma of the breast with silicone granuloma.
\end{abstract}

Key words angiosarcoma of the breast, silicone granuloma, augmentation, mammoplasty

\section{INTRODUCTION}

Angiosarcoma of the breast is a rare non-epithelial tumor that accounts for less than $0.1 \%$ of primary malignancies of the breast [1]. Moreover, there has been only one report, by Cuesta et al. of the development of angiosarcoma of the breast in a patient who underwent breast augmentation with silicone implants [2]. Here we report a case of angiosarcoma with silicone granuloma, and discuss the progress of the disease in detail.

\section{CASE REPORT}

An 87-year-old woman had undergone bilateral breast augmentation with silicone injection in her youth. Although she became aware of a tumor in her right breast, she waited 8 years before seeking treatment. She underwent a medical examination at our hospital, presenting with swelling and pain in the right breast. The mass was palpable, very hard, and $7 \mathrm{~cm}$ in diameter. The cytological findings from fine-needle biopsy showed only blood, however, and she decided to leave the mass untreated. Two years later she came to our hospital again due to an increase in pain and swelling of the tumor, which by that time had grown to over $11 \mathrm{~cm}$ in diameter, along with bleeding. Although we scheduled her for hospitalization, 8 days later, she was brought to our hospital prior to that time by ambulance for continuous bleeding from the tumor.

Her medical history included left breast mastitis, a past healed fracture in the right femur, and a right cataract operation, but no history of radiation exposure. Her family history was unremarkable.

Corresponding author: Miki Takenaka, Department of Surgery, Kurume University School of Medicine, 67 Asahi-machi, Kurume 830-0011, Japan. Tel: 0942-31-7566 Fax: 0942-34-0709 E-mail: takenaka_miki@kurume-u.ac.jp

Abbreviations: CD, cluster of differentiation; HE, hematoxylin and eosin stain; MRI, magnetic resonance imaging; US, ultrasonography. 
Laboratory data were as follows: red blood cell count, $254 \times 10^{4} / \mu 1$; hemoglobin, $7.6 \mathrm{~g} / \mathrm{dl}$; and C-reactive protein, $5.35 \mathrm{mg} / \mathrm{dl}$, indicating anemia and systemic inflammation. Physical findings included hardened silicone in the bilateral breasts. The skin on her

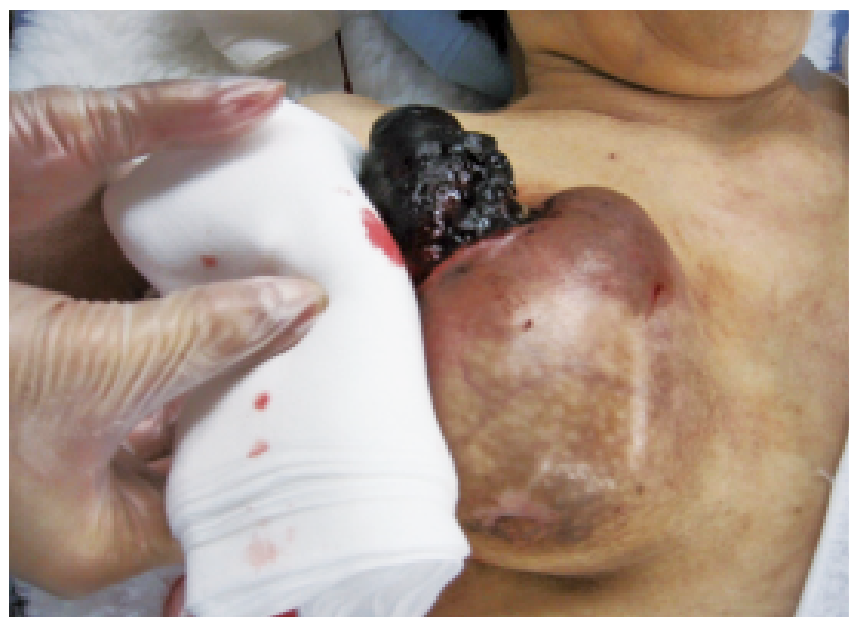

Fig. 1. The tumor had dark purple and tough skin with hematoma. right breast was dark purple and tough, with a large tumor $11 \mathrm{~cm}$ in diameter with hematoma and marked protrusion (Fig. 1). Chest X-ray appeared normal, but mammography was impossible due to the tumor. Ultrasonography (US), showed a mass with solid com-

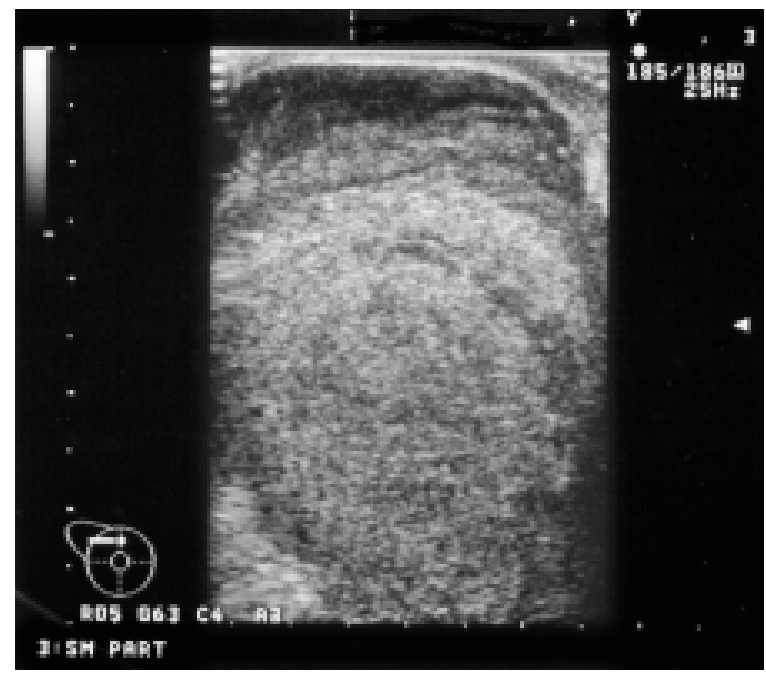

Fig. 2. US findings showed a solid mass, with low echoic area of bloody liquid.

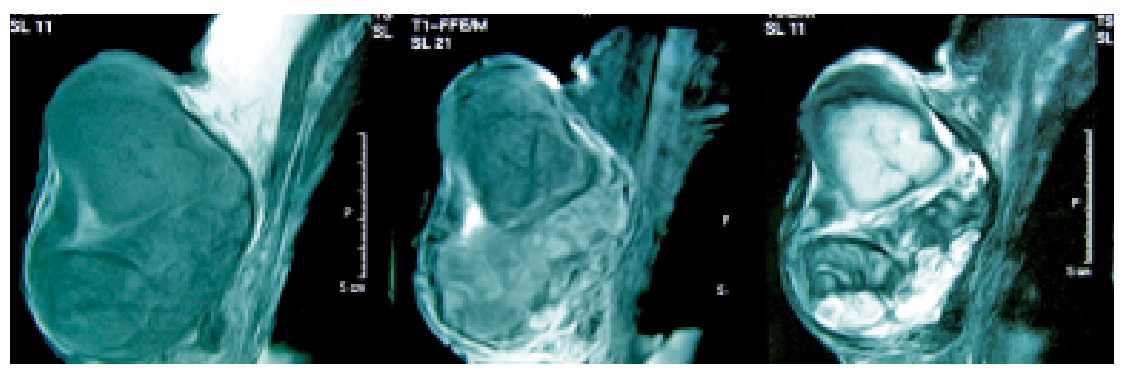

Fig. 3. MRI findings showed a mass $10.5 \times 8 \times 7 \mathrm{~cm}$, with slight enhancement at the edge of the lower outer quadrant of the right breast in the dynamic study.

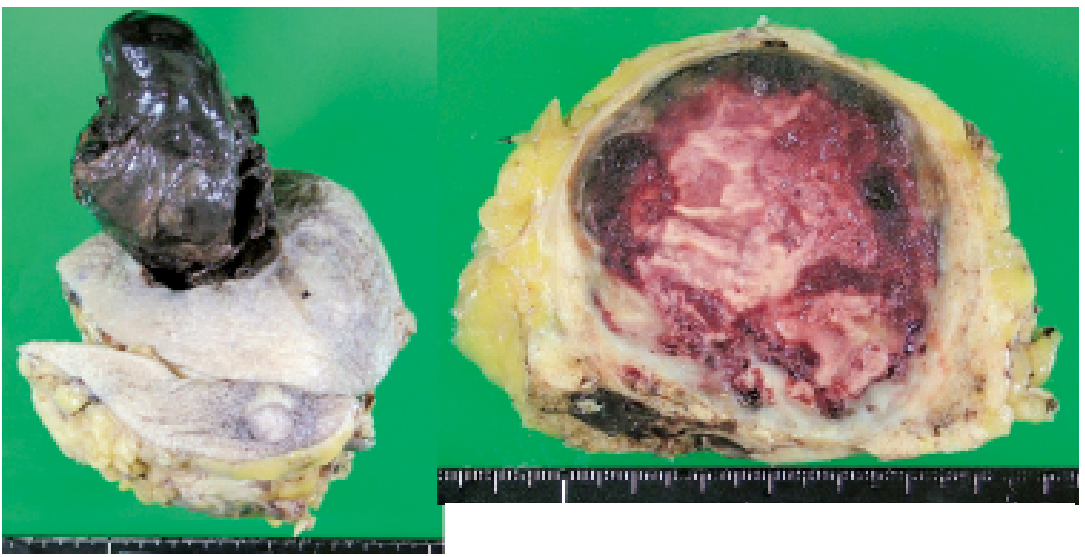

Fig. 4. Macroscopically, the tumor was $7.5 \times 7.5 \mathrm{~cm}$ in size, and had a central solid pinkish area with hematoma and necrosis in parts. 
ponents in her right breast. The mass included a low echoic area, which suggested that part of the tumor was filled with bloody liquid due to necrosis (Fig. 2). Magnetic resonance imaging (MRI) showed a mass $10.5 \times 8 \times 7 \mathrm{~cm}$ in size, and the edge of the lower outer quadrant of the right breast was enhanced slightly on the dynamic MRI. An area of high intensity in T2 weighted MRI suggested hematoma in the upper outer quadrant of the right breast (Fig. 3).

After a systemic examination, she was transfused for anemia. We tried to stop the bleeding by pressure with epinephrine solution gauze, but the bleeding continued. We then scheduled surgery after systemic stabilization taking into account her advanced age of 87 years. However, a projectile hemorrhage from her tumor started on the 4th day of hospitalization, and we immediately performed an emergency mastectomy. The total blood loss was $2170 \mathrm{cc}$ during the 4 days just prior to and during the operation.

Macroscopically the tumor was $7.5 \times 7.5 \mathrm{~cm}$ in size, with a central solid pinkish area with hematoma and partial necrosis (Fig. 4). Histologically, the central

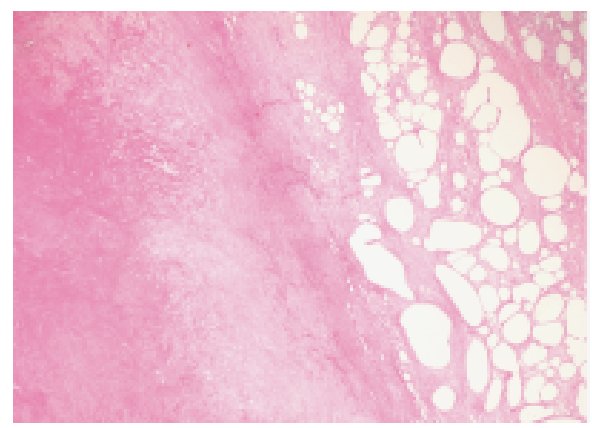

a

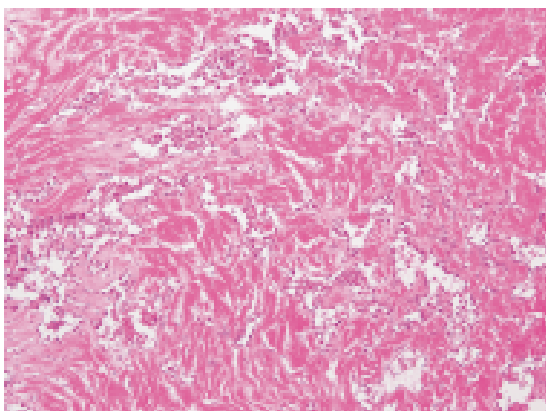

b

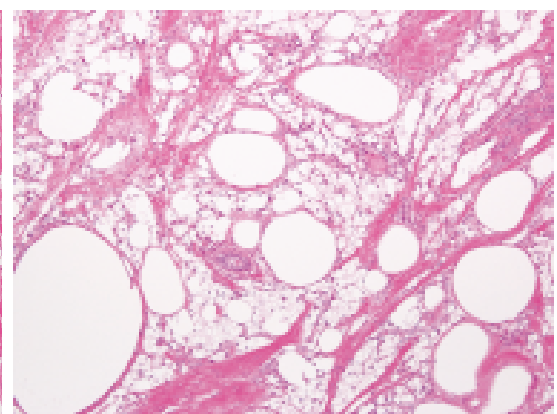

C

Fig. 5. Microscopic findings. (a): The central area of the tumor showed cellular degeneration (left), and the surrounding area showed silicone granuloma (right). Tumor cells were seen between regions of degeneration and granuloma (HE, low magnification). (b): The proliferation of neoplastic endothelial cells (HE, high magnification). (c): Silicone granuloma (HE, high magnification).

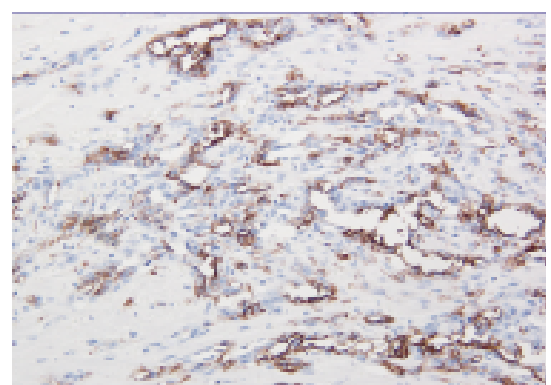

a

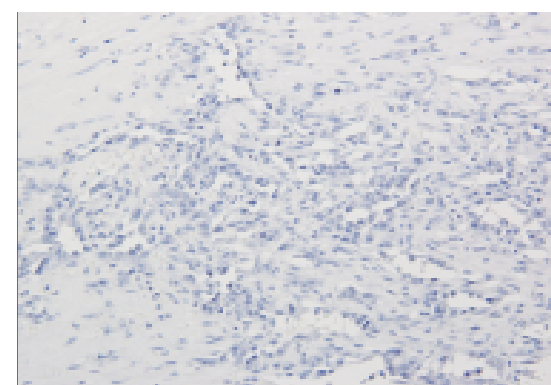

b

Fig. 6. On immunostaining, the neoplastic endothelial cells were positive for CD34 (Fig. 6a), and negative for CAM5.2 (Fig. 6b). 
area of the tumor showed degeneration and necrosis, and the surrounding area consisted of silicone granuloma. Little viable tissue was present between the degeneration and the granuloma (Fig. 5a). The tumor cells caused expansion in papillary vascular channels, covered with neoplastic endothelial cells (Fig. 5b), and were grade II using Rosen's 3-tier system [3]. The granuloma was seen partly within the silicone and was surrounded with hyalinization and fibrosis (Fig. 5c). Immunohistochemically, the neoplastic endothelial cells were positive for cluster of differentiation (CD) 34 and negative for cytokeratin (CAM5.2) (Fig. 6a and $6 b)$.

Two months after the operation, she died of rapid lung metastasis, carcinomatous pleural effusion, and pneumonia.

\section{DISCUSSION}

Angiosarcoma of the breast is a rare entity that accounts for less than $0.1 \%$ of primary mammary malignancy [1]. The disease has a relatively higher occurrence among younger persons, with the average age of patients standing at 36.9 years in a range from 19 to 79 years [4].

The clinical symptoms can involve the sensation of a soft, painless mass that exhibits rapid growth. It can also involve discoloration of the skin and bleeding, as in the present case. Preoperative diagnosis of the disease is difficult, since the specimens for cytodiagnosis appear with abundant blood in the background, and angiosarcoma of the breast is often mistakenly diagnosed as benign hemangioma after fine needle aspiration biopsy. It has been reported that immunostaining is effective as an auxiliary diagnostic tool [4-6]. Due to the paucity of characteristic features in imaging tests, the average duration from the first medical examination to established diagnosis is quite long (11 months), and the average tumor is large in diameter $7.6 \mathrm{~cm} \mathrm{[4].}$

The treatment of first choice is surgical resection. Metastasis occurs mainly by hematogenous means, accordingly lymph node dissection is not necessarily required.

The prognosis is generally extremely poor, but chemotherapy (adriamycin, cisplatin, ifosfamide and paclitaxel combination [7], or weekly paclitaxel [8]) and interleukin- 2 treatment has been reported to be effective $[9,10]$. The average survival period is 22 months with a three-year survival rate of $38 \%$ [11]. The tumor diameter is the most important prognostic factor. The five-year survival rate is $70 \%$ if the tumor diameter is less than $5 \mathrm{~cm}$, but only $35 \%$ if the tumor diameter is more than $5 \mathrm{~cm}$, with no significant effect from tumor grade on survival rate [12].

The relationship between breast augmentation and angiosarcoma of the breast remains unclear. The angiosarcoma and silicone granuloma in this case may have been unrelated. There has been a report, however, by Cuesta et al. on the development of angiosarcoma of the breast in a patient who underwent breast augmentation with silicone implants [2]. Multiple myeloma after silicone breast implants [13], and cutaneous T-cell lymphoma after silicone breast implants [14], has been reported in the literature. The relationship remains unclear, but T-cell-mediated autoimmune reactions may have some relationship with silicone breast implants [14]. In Japan, there have been 40 cases of breast cancer that may have been caused by breast augmentation. However, no correlation has been discovered between cancer and breast augmentation in experimental studies, and clinical case reports in the United States and Europe have also been unable to establish a correlationship [15].

In recent years, breast implants have become fashionable since a wider variety of breast augmentation methods have become available, including silicone infusion, silicone bag implant, normal saline solution bag implant, and hyaluronic acid infusion. In part the increase in breast implants may be attributed to changes in women's values and concepts of beauty. However, in many cases, diagnosis of neoplasm of the breast after breast augmentation is difficult. Therefore, we ought to take into consideration the risks from asyet-unknown foreign-body reactions and complicating factors.

ACKNOWLEDGMENTS: We thank Professor Hiroshi Hashimoto, of the Department Pathology, University of Occupational and Environmental Health, for kindly reading an earlier draft.

\section{REFERENCES}

1. Tsumagari K, Sakamoto G, Akiyama F, Makita M, Takahashi K et al. 3 cases of angiosarcoma of the breast. Jpn J Breast Cancer 2001; 16:359-364. (in Japanese)

2. Cuesta T, Leon B, Abel J, and Valenzuela J. Breast angiosarcoma in a patient with multi-surgery and mammary implants. Ginec Obst Mex 2002; 70:76-81.

3. Rosen PP, Kimmel M, and Ernsberger D. Mammary angiosarcoma/ The prognostic significance of tumor differentiation. Cancer 1988; 62:359-364.

4. Nakada T, Kashizuka T, Itou T, Tsuchiya J, and Nawa M. A case of mammary angiosarcoma. Jpn J Clin Surgery 2004; 65:2086-2089. (in Japanese) 
5. Gherardi G, Rossi S, Perrone S, and Scanni A. Angiosarcoma after breast-conserving therapy. Cancer Cytopathol 2005; 105:145-151.

6. Yoshida K, Yagi S, Yamada T, Kitagawa S, Nakagawa M et al. A case of angiosarcoma of the breast surviving without recurrence for 5 years after breast conserving surgery. Jpn J Clin Surgery 2000; 61:3210-3213. (in Japanese)

7. Asmane I, Lituque V, Heymann S, Marcellin L, Cecile A et al. Adriamycin, Cisplatin, Ifosfamide and Paclitaxel Combination as Front line Chemotherapy for Locally Advanced and Metastatic Angiosarcoma. Anticancer Research 2008; 28:3041-3046.

8. Penel N, Bui BN, Bay JO, Cupissol D, Ray-Coquard I et al. Phase II trial of weekly paclitaxel for unresectable angiosarcoma. J Clin Oncol 2008; 26:5269-5274.

9. Tanaka K, Sonoo H, Yamamoto Y, Utagawa K, Arime I et al. A case of angiosarcoma of the breast and a review of the Japanese literature. Jpn J Breast Cancer 1999; 14:93-98. (in Japanese)

10. Nakayama S, Oomori H, Takeda H, Takubo K, Tai M et al. A case of angiosarcoma of the breast with recurrence in the other breast at 3 years post-operatively. Jpn J Clin Surgery 2004; 65:2090-2094. (in Japanese)

11. Chen T, Kirkegaard D, and Bocian J. Angiosarcoma of the breast. Cancer 1980; 46:368-371.

12. Vorburger S, Xing Y, Hunt K, Lakin G, Benjamin R et al. Angiosarcoma of the breast. Cancer 2005; 104:2682-2688.

13. Garland L, Ballester F, Vasey B, Benson K, Moscinski C et al. Multiple myeloma in women with silicone breast implants. Curr Top Microbiol Immunol 1996; 210:361-366.

14. Duvic M, Moore D, Menter A, and Vonderheid C. Cutaneous T-cell lymphoma in association with silicone breast implants. J Am Acad Dermatol 1995; 32:939-942.

15. Ogasawara Y, Ohsumi S, Saeki T, and Takashima S. 3 cases of breast cancer after breast augmentation. Jpn J Breast Cancer 1998; 13:834-838. (in Japanese) 